\title{
Assistência de enfermagem aos pacientes com Doença de Alzheimer em cuidados paliativos: revisão sistemática
}

\author{
Nursing care for patients with Alzheimer's disease in palliative care: a systematic review \\ Atención de enfermería a pacientes con enfermedad de Alzheimer en cuidados paliativos: \\ una revisión sistemática
}

Tânia Maria Rocha Guimarães ${ }^{2 *}$, Karla Naiara França Silva ${ }^{1}$, Heloíza Gabrielly de Oliveira Cavalcanti', Ingridy Christian Araújo de Souza ${ }^{1}$, Jacqueline dos Santos Leite ${ }^{1}$, Jeovanna Thamires Bezerra da Silva ${ }^{1}$, Júlia Rebeka de Lima ${ }^{1}$, Karoline Lupicinio de Andrade ${ }^{1}$, Fábia Maria de Lima ${ }^{1}$.

\section{RESUMO}

Objetivo: Realizar uma revisão sistemática de literatura sobre os cuidados de enfermagem ao paciente com doença de Alzheimer em cuidados paliativos. Métodos: Estudo de revisão sistemática conduzido conforme a metodologia Preferred Reporting Items for Systematic Reviews and Meta-Analyses (PRISMA). O recorte temporal foi realizado no período de cinco anos (2013 a 2017), nos idiomas inglês, português e espanhol. Foram utilizadas como fontes de informação: Biblioteca Virtual de Saúde, MEDLINE, LILACS, BDENF e os descritores "doença de Alzheimer", combinados com "cuidados paliativos" e "enfermagem", utilizando o operador booleano "AND". Resultados: Verificamos escassez de publicações sobre o tema. Dos 48 artigos selecionados, apenas 6 atenderam os critérios de inclusão. Verificamos cinco temas principais: diálogo aberto com o paciente e família; cuidado integral realizado por equipe multidisciplinar de forma humanizada; o papel do enfermeiro; alívio do sofrimento e da dor para melhorar a qualidade de vida; a importância da abordagem espiritual. Conclusão: A assistência de enfermagem deve ser realizada de forma integral e humanizada, atendendo as necessidades físicas, psicológicas e espirituais do paciente com doença de Alzheimer e de seus familiares.

Palavras-chave: Cuidados paliativos, Doença de Alzheimer, Enfermagem, Envelhecimento.

\begin{abstract}
Objective: Conduct a systematic review of the literature on nursing care for patients with Alzheimer's disease in palliative care. Methods: Systematic review study conducted according to the Preferred Reporting Items for Systematic Reviews and Meta-Analyzes (PRISMA) methodology. The time clipping was carried out over a five-year period (2013-2017), in English, Portuguese and Spanish. The following sources of information were used: Virtual Health Library, MEDILINE, LILACS, BDENF and the descriptors "Alzheimer's disease", combined with "palliative care" and "nursing" using the boolean operator "AND". Results: We found a shortage of publications on the subject. Of the 48 articles selected, only 6 met the inclusion criteria. We analyzed five main themes: open dialogue with patient and Family; Comprehensive care provided by a multidisciplinary team in a humanized manner; the role of the nurse; relief from suffering and pain to improve quality of life; the importance of the spiritual approach. Conclusion: Nursing care must be performed in its entirety and humanized manner, meeting the physical, psychological and spiritual needs of patients with Alzheimer's disease and their families.
\end{abstract}

Keywords: Palliative Care, Alzheimer's disease, Nursing, Aging.

\footnotetext{
1 Universidade de Pernambuco (UPE) - FENSG, Recife-PE.

${ }^{2}$ Faculdade de Enfermagem Nossa Senhora das Graças (FENSG) Recife-PE.

*E-mail: tmrguimaraes@gmail.com
} 


\section{RESUMEN}

Objetivo: realizar una revisión sistemática de la literatura sobre cuidados de enfermería para pacientes con enfermedad de Alzheimer en cuidados paliativos. Métodos: Estudio revisión sistemática conducida conforme a la metodología Preferred Reporting Items for Systematic Reviews y Meta-Analys (PRISMA). El recorte de tiempo se realizó durante un período de cinco años (2013-2017), en inglés, portugués y español. Se utilizaron las siguientes fuentes de información: Biblioteca Virtual en Salud, MEDLINE, LILACS, BDENF y los descriptores "Enfermedad de Alzheimer", combinados con "cuidados paliativos" y "enfermería", utilizando el operador booleano "Y". Resultados: Verificamos la escasez de publicaciones sobre el tema. De los 48 artículos seleccionados, solo 6 cumplieron los criterios de inclusión. Analizamos cinco temas: diálogo abierto con el paciente y la familia; atención integral realizada por un equipo multidisciplinario de manera humanizada; el papel de la enfermera; alivio del sufrimiento y el dolor para mejorar la calidad de vida; La importancia del enfoque espiritual. Conclusión: La atención de enfermería debe realizarse de manera integral y humanizada, satisfaciendo las necesidades físicas, psicológicas y espirituales de los pacientes con enfermedad de Alzheimer y sus familias.

Palabras clave: Cuidados Paliativos, Enfermedad de Alzheimer, Enfermería, Envejecimiento.

\section{INTRODUÇÃO}

O envelhecimento de uma população caracteriza-se pelo aumento na quantidade de pessoas com idade maior de 60 anos e a diminuição no número de crianças e jovens. Populações em todo o mundo estão envelhecendo rapidamente. A proporção de pessoas idosas está crescendo mais rapidamente que a de qualquer outra faixa etária. Entre 1970 e 2025, espera-se um crescimento de $223 \%$, ou em torno de 694 milhões, no número de pessoas mais velhas. Em 2025, existirá um total de aproximadamente 1,2 bilhões de pessoas idosas. Até 2050 haverá 2 bilhões, sendo 80\% nos países em desenvolvimento (WHO, 2015).

Estimativas para 2025 preveem que o Brasil ocupará o sexto lugar mundial com 32 milhões de pessoas idosas. Já em 2060 , os idosos representarão $33,7 \%$ da população total (WHO, 2015). A redução da taxa de fecundidade, da taxa de mortalidade infantil e o aumento da expectativa de vida são determinantes da transição demográfica brasileira. Em função dessa mudança populacional, o país terá um elevado número de idosos com doenças crônico-degenerativas, e dentro dessa classe estão as demências (SANTOS FH, et al.,2018).

Demência é uma síndrome devida à doença cerebral, de natureza crônica e progressiva, na qual há um comprometimento de funções corticais, incluindo memória, raciocínio, orientação, compreensão, cálculo, capacidade de aprendizagem, linguagem e julgamento. Com o comprometimento dessas funções corticais. A demência pode trazer modificações na qualidade de vida e na capacidade funcional dos indivíduos que apresentam essa doença (ALZHEIMER'S ASSOCIATION, 2018).

A mais comum das síndromes demenciais é a doença de Alzheimer (DA). O acúmulo do fragmento de proteína beta-amiloide fora dos neurônios dando origem às placas senis e a hiperfosforilação da proteína TAU nos neurônios, levando a formação de emaranhados neurofibrilares são duas das várias alterações cerebrais associadas à doença. Acredita-se que as placas beta-amiloides contribuam para a morte celular, interferindo na comunicação neurônio-neurônio nas sinapses, enquanto os emaranhados de tau bloqueiam o transporte de nutrientes e outras moléculas essenciais dentro dos neurônios. À medida que a quantidade de betaamiloide aumenta, atinge-se um ponto de inflexão no qual a tau anormal se espalha pelo cérebro (NIH e NIA, 2019).

Habitualmente, a doença é de evolução lenta e pode afetar o indivíduo de diferentes maneiras. O padrão mais comum de sintomas começa de forma insidiosa, com a piora gradual da memória, acompanhada de dificuldades na apreensão de novas informações e perda da habilidade de realizar tarefas da vida diária. À medida que a doença evolui, a deterioração é progressiva e as pessoas experimentam dificuldades no 
gerenciamento da sua vida, o que as torna dependentes de ajuda para a realização de simples tarefas do dia a dia. Na fase avançada, além do comprometimento da memória remota, ocorre a necessidade de supervisão para atividades básicas como tomar banho, se vestir, ir ao banheiro, comer e outros afazeres da vida diária, além de alterações comportamentais como irritabilidade, agressividade e alucinações. Na fase final da doença a pessoa perde a capacidade de se comunicar, deixa de reconhecer seus familiares e amigos, fica restrita ao leito e dependente de cuidados permanentes em horário integral (ALZHEIMER'S ASSOCIATION, 2018).

Sendo assim, o cuidado paliativo (CP) é visto como ferramenta importante na prática assistencial para que dessa forma a equipe multiprofissional proporcionem uma assistência de qualidade, de forma holística, humanizada e singular no tratamento ao paciente terminal de DA (QUEIROZ RB, et al., 2014).

Dessa forma, o CP é uma tarefa complexa e desafiadora, sendo adotada na modernidade para os cuidados necessários no final da vida, que representam uma alternativa contemporânea da morte. Nesse âmbito, o ponto de vista terapêutico é voltado para a qualidade de vida (WHO, 2017). Os cuidados não possuem a finalidade de curar, uma vez que a doença já se encontra em um estágio progressivo, irreversível e não responsivo ao tratamento curativo, sendo o objetivo desses cuidados proporcionar qualidade de vida nos momentos finais (JOHNSON C, 2017).

Assim, é importante reafirmar a importância da vida, considerando a morte como um processo natural, estabelecendo um cuidado que oferece alívio da dor e de outros sintomas, integrando os aspectos psicológicos e espirituais, para que a família possa enfrentar a doença e sobreviver ao período de luto (ARAÚJO MMT e SILVA MJP, 2007).

Nesse contexto, compete aos enfermeiros entender os desejos da família, levar em consideração a posição e a circunstância desse ambiente familiar, o suporte de que o paciente necessitará, quanto à terapêutica e aos cuidados de enfermagem, assim como um cuidar holístico, humanizado e singular de um paciente em cuidados paliativos (SILVA RS, et al., 2011; JOHNSON C, 2017).

Diante do exposto, definiu-se como questão norteadora: Como os profissionais de enfermagem atuam no cuidado à pessoa com DA em conduta paliativa? Assim, o objetivo do estudo foi identificar os cuidados de enfermagem ao paciente com doença de Alzheimer em cuidados paliativos.

\section{MÉTODOS}

Trata-se de um estudo de revisão sistemática, conduzido conforme a metodologia Preferred Reporting Items for Systematic Reviews and Meta-Analyses (PRISMA) (MOHER et al.,2009). A coleta de dados foi realizada em maio de 2018, a partir das bases de dados on-line LILACS (Literatura Latino-Americana e do Caribe em Ciências da Saúde) e MEDLINE (Literatura Internacional em Ciências da Saúde) e BDENF (Base de dados de enfermagem), contidos na Biblioteca Virtual de Saúde (BVS).

Os descritores em saúde selecionados foram: Cuidados Paliativos and Doença de Alzheimer; e Cuidados Paliativos and Enfermagem and Doença de Alzheimer. Todos os processos de seleção e avaliação de artigos foram realizados por pares. Os filtros selecionados para busca dos descritores foram: 1. Idiomas (inglês e português); 2. Ano de publicação (2013-2017); 3. Tipo de documento (artigos). Foram excluídas as crônicas, narrativas, cartas ao editor, dissertações teses e artigos duplicados e sem resumo.

Uma análise inicial foi realizada com base nos títulos e resumos de todos os artigos que preenchiam os critérios de inclusão. Após análise dos resumos, todos os artigos selecionados foram obtidos na íntegra e posteriormente examinados.

\section{RESULTADOS}

Para os descritores Cuidados Paliativos and Doença de Alzheimer, foram achados 38 artigos, e para Cuidados Paliativos and Enfermagem and Doença de Alzheimer foram achados 10 artigos, totalizando 48 artigos, descritos na Tabela 1. 
Tabela 1 - Número de artigos selecionados sobre os cuidados de enfermagem ao paciente com doença de Alzheimer em conduta paliativa, selecionados conforme cruzamento dos descritores, segundo a Base de Dados, período 2013-2017.

\begin{tabular}{ccc}
\hline CRUZAMENTO DE DESCRITORES & BASE DE DADOS & RESULTADOS \\
\hline Cuidados Paliativos and Doença de & MEDLINE & 34 \\
Alzheimer & LILACS & 3 \\
BDENF & & \\
$\begin{array}{c}\text { Cuidados Paliativos and Enfermagem } \\
\text { and Doença de Alzheimer }\end{array}$ & MEDLINE & 10 \\
\hline Total & & 48 \\
\hline
\end{tabular}

Fonte: Guimarães TMR, et al., 2018.

Dos 38 artigos selecionados com os descritores Cuidados Paliativos and Doença de Alzheimer, 7 não tinham texto completo disponível, 4 estavam indisponíveis de forma gratuita, e 3 foram encontrados em duplicidade, desta forma, 24 artigos estavam disponíveis de forma completa e gratuita, porém, 12 não abordavam o tema proposto e 6 não eram artigos, com isso, aplicados os critérios de inclusão foram selecionados 6 artigos.

E para os descritores Cuidados Paliativos and Enfermagem and Doença de Alzheimer, dos 10 artigos selecionados, 2 não tinham textos completos disponíveis, 3 estavam indisponíveis de forma gratuita e 5 foram encontrados em duplicidade com os artigos da busca anterior, desta forma, nenhum artigo foi selecionado (Tabela 2).

Tabela 2 - Critérios de exclusão dos artigos selecionados sobre os cuidados de enfermagem ao paciente com doença de Alzheimer em conduta paliativa, período 2013-2017.

\begin{tabular}{|c|c|c|c|}
\hline $\begin{array}{l}\text { CRUZAMENTO } \\
\text { DESCRITORES }\end{array}$ & $\begin{array}{c}\text { CRITÉRIOS DE EXCLUSÃO } \\
\text { ARTIGOS }\end{array}$ & $\begin{array}{l}\text { ARTIGOS NÃO } \\
\text { SELECIONADOS }\end{array}$ & $\begin{array}{c}\text { ARTIGOS } \\
\text { SELECIONADOS }\end{array}$ \\
\hline \multirow[t]{2}{*}{$\begin{array}{l}\text { Cuidados Paliativos } \\
\text { And Doença de } \\
\text { Alzheimer }\end{array}$} & $\begin{array}{l}\text { Não abordavam o tema proposto } \\
\text { Sem textos completos disponíveis } \\
\text { Não se enquadra como artigo } \\
\text { Indisponíveis de forma gratuita } \\
\text { Duplicidade }\end{array}$ & $\begin{array}{c}12 \\
7 \\
6 \\
4 \\
3\end{array}$ & 6 \\
\hline & Total & 32 & \\
\hline $\begin{array}{l}\text { Cuidados Paliativos } \\
\text { And Enfermagem And } \\
\text { Doença de Alzheimer }\end{array}$ & $\begin{array}{l}\text { Artigos em duplicidade } \\
\text { Indisponíveis de forma gratuita } \\
\text { Textos completos indisponíveis }\end{array}$ & $\begin{array}{l}5 \\
3 \\
2\end{array}$ & 0 \\
\hline Doença de Alzheimer & Total & 10 & \\
\hline
\end{tabular}

Fonte: Guimarães TMR, et al., 2018.

Depois de aplicados os filtros, 48 artigos foram encontrados nas bases de dados, porém, a amostra final desta revisão foi constituída por 6 artigos científicos, selecionados pelos critérios de exclusão previamente estabelecidos e realizada leitura completa dos artigos (Fluxograma 1). 
Fluxograma 1 - Seleção dos artigos para análise científica sobre os cuidados de enfermagem ao paciente com doença de Alzheimer em cuidados paliativos, 2013-2017.

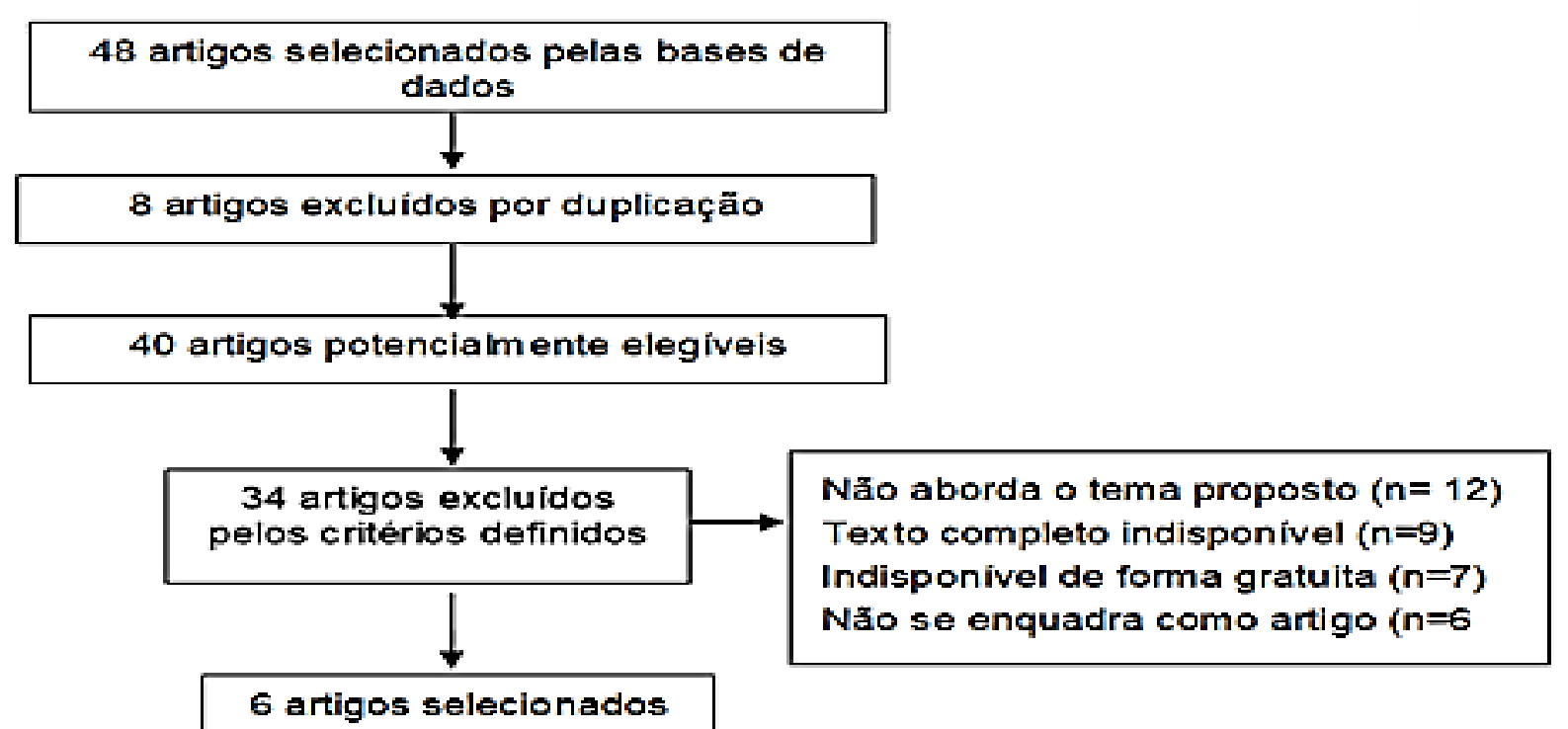

Fonte: Guimarães TMR, et al., 2018.

Os seis artigos selecionados foram organizados de acordo com autor/autores, o ano de publicação, tipo do estudo, revista, e base de dados, conforme descrito no Quadro 1.

Verificamos que há uma predominância de artigos na modalidade Revisão de Literatura $3(50 \%)$ e que a maioria dos estudos são internacionais 5 (83\%) e foi encontrada na base de dados MEDLINE 5 (83\%).

Quadro 1 - Artigos sobre os cuidados de enfermagem ao paciente com doença de Alzheimer em conduta paliativa, selecionados organizados por autor, ano, título, tipo de estudo, revista e base de dados.

\begin{tabular}{|l|c|l|c|c|c|}
\hline \multicolumn{1}{|c|}{ Autores } & Ano & \multicolumn{1}{|c|}{ Título } & $\begin{array}{c}\text { Tipo de } \\
\text { estudo }\end{array}$ & Revista & $\begin{array}{c}\text { Base de } \\
\text { dados }\end{array}$ \\
\hline Mitchell SL & 2015 & Advanced Dementia. & $\begin{array}{c}\text { Descritivo } \\
\text { Qualitativo }\end{array}$ & N Engl J Med & MEDLINE \\
\hline $\begin{array}{l}\text { Volicer L, } \\
\text { Simard J }\end{array}$ & 2015 & $\begin{array}{l}\text { Palliative care and quality of } \\
\text { life for people with dementia: } \\
\text { medical and psychosocial } \\
\text { interventions. }\end{array}$ & $\begin{array}{l}\text { Revisão de } \\
\text { Literatura }\end{array}$ & $\begin{array}{l}\text { International } \\
\text { Psychogeriatrics }\end{array}$ & MEDLINE \\
\hline $\begin{array}{l}\text { Queiroz } \\
\text { RB, et al. }\end{array}$ & 2014 & $\begin{array}{l}\text { Cuidados paliativos e } \\
\text { Alzheimer: concepções de } \\
\text { neurologistas. }\end{array}$ & $\begin{array}{l}\text { Descritivo } \\
\text { Qualitativo }\end{array}$ & $\begin{array}{c}\text { Rev. Enfermagem } \\
\text { UERJ }\end{array}$ & LILACS \\
\hline $\begin{array}{l}\text { Hendriks } \\
\text { SA,et al. }\end{array}$ & 2014 & $\begin{array}{l}\text { Dying with dementia: } \\
\text { symptoms, treatment, and } \\
\text { quality of life in the last week } \\
\text { of life. }\end{array}$ & $\begin{array}{l}\text { Estudo } \\
\text { transversal }\end{array}$ & $\begin{array}{c}\text { Journal of Pain and } \\
\text { Symptom } \\
\text { Management }\end{array}$ & MEDLINE \\
\hline $\begin{array}{l}\text { Raymond } \\
\text { M, et al. }\end{array}$ & 2014 & $\begin{array}{l}\text { Palliative and end of life care } \\
\text { for people with dementia: } \\
\text { lessons clinical } \\
\text { commissioners. }\end{array}$ & $\begin{array}{l}\text { Revisão de } \\
\text { Literatura }\end{array}$ & $\begin{array}{c}\text { Primary Health Care } \\
\text { Research \& } \\
\text { Development }\end{array}$ & MEDLINE \\
\hline $\begin{array}{l}\text { Yeaman } \\
\text { PA, et al. }\end{array}$ & 2013 & $\begin{array}{l}\text { Providing quality palliative } \\
\text { care in end-stage Alzheimer } \\
\text { disease. }\end{array}$ & $\begin{array}{l}\text { Revisão de } \\
\text { Literatura }\end{array}$ & $\begin{array}{l}\text { American Journal of } \\
\text { Hospice \& Palliative } \\
\text { Medicine® }\end{array}$ & MEDLINE \\
\hline
\end{tabular}

Fonte: Guimarães TMR, et al., 2018. 
Os resultados que trazem os artigos selecionados estão descritos no Quadro 2.

Quadro 2 - Resultado dos artigos selecionados sobre os cuidados de enfermagem ao paciente com doença de Alzheimer em conduta paliativa, 2013-2017.

\begin{tabular}{|c|c|}
\hline Título do artigo & $\begin{array}{l}\text { Assistência prestada ao paciente com Alzheimer em cuidados } \\
\text { paliativos }\end{array}$ \\
\hline 1-Advanced Dementia & $\begin{array}{l}\text { - Educar os pacientes e familiares sobre a doença, } \\
\text { - } \text { Envolver os pacientes e famílias no planejamento da assistência. } \\
\text { - Promorar cuidado de acordo com as preferências do paciente. } \\
\text { - As metas de atenções e tratamento para sintomas angustiantes. }\end{array}$ \\
\hline $\begin{array}{l}\text { 2-Palliative care and } \\
\text { quality of life for people } \\
\text { with dementia: medical } \\
\text { and psychosocial } \\
\text { interventions }\end{array}$ & $\begin{array}{l}\text { - Notificar o diagnóstico e as consequências para a vida futura do } \\
\text { indivíduo e assim deixá-lo tomar decisões de fim de vida. } \\
\text { - Monitorar sinais não-verbais de dor. } \\
\text { - Envolver o paciente em atividades que lhes interessam. } \\
\text { - Prover assistência psicológica e espiritual. } \\
\text { - O cuidado pode mudar caso o paciente rejeite. }\end{array}$ \\
\hline $\begin{array}{l}\text { 3-Cuidados paliativos e } \\
\text { Alzheimer: concepções } \\
\text { de neurologistas }\end{array}$ & $\begin{array}{l}\text { - Entender os desejos da família quanto à terapêutica e aos cuidados de } \\
\text { enfermagem, assim como um modo de cuidar holístico e humanizado. } \\
\text { - Aliviar a dor, proporcionar suporte psicossocial e espiritual, valorizar o } \\
\text { conforto, o bem-estar, o zelo, a dedicação, a empatia e a atenção. } \\
\text { - O controle e o alívio da dor são um direito do indivíduo e um dever dos } \\
\text { profissionais, que devem criar estratégias para diminuir o sofrimento. } \\
\text { - Sintomas psicológicos e comportamentais podem ser tratados com } \\
\text { terapia, estimulação multissensorial e cognitiva, exercícios físicos, } \\
\text { musicoterapia, recreação e arteterapia. } \\
\text { - O cuidado deve ser pautado na escuta, na percepção, na compreensão } \\
\text { e na identificação das necessidades para, só então, planejar ações. }\end{array}$ \\
\hline $\begin{array}{l}\text { 4-Dying with dementia: } \\
\text { symptoms, treatment, } \\
\text { and quality of life in the } \\
\text { last week of life }\end{array}$ & $\begin{array}{l}\text { - O controle dos sintomas é um fator importante na melhoria da qualidade } \\
\text { de vida do paciente. } \\
\text { - Uso medidas não-farmacológicas para o alívio da dor (fisioterapia, } \\
\text { terapêutica ocupacional, neuro-estimulação eléctrica, massagem). }\end{array}$ \\
\hline $\begin{array}{l}\text { 5-Palliative and end of life } \\
\text { care for people with } \\
\text { dementia: lessons for } \\
\text { clinical commissioners. }\end{array}$ & $\begin{array}{l}\text { - Melhor comunicação entre cuidadores e pessoas com demência pode } \\
\text { - } \quad \text { Planentar o bem-estar dos pacientes. } \\
\text { dor e educação da beneficiaiam os cuidados paliativos. } \\
\text { - Tratamentos invasivos devem ser desencorajados. } \\
\text { - Comunicação, relações sociais positivas e ambientes de cuidados e de } \\
\text { apoio diminuem explosões de agitação e agressividade. }\end{array}$ \\
\hline $\begin{array}{l}\text { 6-Providing quality } \\
\text { palliative care in end- } \\
\text { stage Alzheimer disease. }\end{array}$ & $\begin{array}{l}\text { - Os profissionais de saúde precisam discutir as decisões de fim de vida } \\
\text { com os pacientes, cuidadores e familiares logo após o diagnóstico e } \\
\text { início do tratamento. } \\
\text { - Permitir que pacientes com doenças terminais experimentar períodos } \\
\text { de lucidez e permitir a conectividade com a família como forma de } \\
\text { melhorar a qualidade da vida, preservando a dignidade. } \\
\text { - Os cuidados paliativos melhoram a qualidade de vida e alivia o } \\
\text { sofrimento. }\end{array}$ \\
\hline
\end{tabular}

Fonte: Guimarães TMR, et al., 2018. 


\section{DISCUSSÃO}

O cuidado paliativo preza pela qualidade de vida tanto para o paciente enfermo quanto para a sua família. O Sistema Único de Saúde (SUS) tem um papel decisivo na promoção dessa assistência paliativa humanizada. A organização dos cuidados paliativos deverá ter como objetivo integrar os cuidados paliativos na rede de atenção à saúde (BRASIL, 2018).

A resolução № 41, DE 31 DE OUTUBRO DE 2018 normatiza que o SUS deve ofertar gratuitamente cuidados paliativos como parte dos cuidados continuados incluído no âmbito do sistema de saúde, o qual diz respeito ao tratamento e cuidados destinados a toda pessoa afetada por uma doença que ameace a vida, seja aguda ou crônica, a partir do diagnóstico desta condição (BRASIL, 2018):

"Cuidados paliativos consistem na assistência promovida por uma equipe multidisciplinar, que objetiva a melhoria da qualidade de vida do paciente e seus familiares, diante de uma doença que ameace a vida, por meio da prevenção e alívio do sofrimento, da identificação precoce, avaliação impecável e tratamento de dor e demais sintomas físicos, sociais, psicológicos e espirituais"

A partir da leitura e análise crítica dos estudos, puderam-se identificar quatro temas principais que estão ligados, de forma direta ou indireta, aos cuidados de enfermagem voltados ao binômio o idoso com DA e família, sendo divididos nas seguintes categorias:

\section{Diálogo aberto com o paciente e família sobre os objetivos do cuidado}

Os princípios dos cuidados paliativos se aplicam desde o momento do diagnóstico de DA ou de outras doenças degenerativas progressivas. É importante informar o indivíduo que recebeu o diagnóstico sobre a natureza do diagnóstico e as consequências para sua vida futura. O diagnóstico permite o início precoce do tratamento, que pode atrasar a progressão da doença. As pessoas com esse diagnóstico também podem tomar decisões de fim de vida, quando ainda conseguem entender e avaliar os benefícios dos tratamentos médicos quando a demência progride para um estágio avançado (VOLICER L e SIMARD J, 2015).

Portanto, a pessoa diagnosticada com demência irreversível deve ser informada sobre a probabilidade de ser incapaz de tomar decisões sobre si mesma no futuro. Portanto, eles devem ser incentivados a planejar com antecedência suas questões financeiras, local preferido de residência e aceitação de intervenções médicas quando a demência se tornar grave. É importante suscitar preferências da pessoa com DA, porque podem ser diferentes dos desejos dos cuidadores familiares (DENING KH, et al., 2013). Famílias de pessoas com diagnóstico de demência irreversível, como DA, raramente são informados que esta é uma doença terminal e os princípios dos cuidados paliativos não são discutidos com eles. CP é aplicável a partir do momento do diagnóstico da DA, por isso, a maneira como a abordagem aos familiares é realizada guiará os sentimentos e as atitudes que conduzirão pacientes e familiares ao enfrentamento da doença, pois, estes, precisarão tomar várias decisões que virão à tona que direcionarão o binômio saúde e doença (WHO, 2017).

Todos os artigos analisados nesta revisão descreveram a importância de manter o diálogo aberto com o paciente e família sobre os objetivos do cuidado, com ênfase na educação sobre a doença e envolvimento dos pacientes e familiares no planejamento da assistência e prestação do cuidado de acordo com as preferências do paciente (MITCHELL SL, 2015); notificar o diagnóstico e as consequências para a vida futura do indivíduo e assim deixá-lo tomar decisões de fim de vida (VOLICER L e SIMARD J, 2015); os profissionais de saúde precisam discutir as decisões de fim de vida com os pacientes, cuidadores e familiares logo após 0 diagnóstico e início do tratamento (YEAMAN PA, et al.,2013); no início do atendimento é necessário esclarecer a situação clinicado paciente para os familiares e estabelecer o cuidado singular baseado no estado do paciente (RAYMOND M, et al., 2014).

\section{A equipe multidisciplinar, assistência humanizada e o cuidado integral}

Para idosos com patologias crônicas como a DA, a evolução para a morte sobrevém quando o paciente se encontra em condição de fragilidade, com declínio das suas funções biológicas e qualidade de vida. Desse 
modo há uma necessidade de promover assistência integral, não só ao paciente, mas também a seus familiares, pois a aproximação da morte de um ente querido desperta na família um desgaste físico e emocional. Para que se tenha uma assistência integral é necessário que se tenha uma abordagem holística e humanizada aos envolvidos, garantindo conforto e alívios dos sintomas (QUEIROZ RB, et al., 2014).

A abordagem multidisciplinar também é essencial para o cuidado, visando uma assistência mais humanizada no final da vida. A equipe deve estar sintonizada, compartilhando informações e trabalhando cooperativamente, entendendo quais são os objetivos a serem atingidos nesta fase do cuidar (JOHNSON C, 2017). Neste sentido, a equipe deve oferecer um tratamento adequado ao paciente com doença de Alzheimer, resgatando a humanização do processo de morrer, e compreender a morte como parte da vida (CARDOSO $\mathrm{DH}$, et al.,2013).

Desta forma, mais do que habilidades técnicas de tratamento, é fundamental que a equipe de saúde desenvolva uma relação de empatia com os pacientes e familiares, sendo importante ouvir e ser sensível às necessidades dos mesmos, valorizando suas experiências, desenvolvendo uma relação baseada na dignidade e respeito de forma a auxiliá-los no processo de morte (JOHNSON C, 2017).

Considerando a assistência integral na revisão realizada, verificamos que o modo de cuidar deve ser holístico e humanizado; a importância de proporcionar suporte psicossocial e espiritual, valorizar o conforto, o bem-estar, o zelo, a dedicação, a empatia e a atenção ao paciente e familiar; preservar a autoestima do paciente, ter carinho e paciência, incentivo às atividades que estimulem as funções mentais; a importância de prover assistência psicológica, espiritual e a prestação do cuidado deve ser de acordo com as preferências do paciente (QUEIROZ RB, et al., 2014; VOLICER L e SIMARD J, 2015).

\section{0 papel do enfermeiro na equipe}

Os cuidados paliativos devem ser desenvolvidos integralmente, desde o controle de sintomas, alívio da dor e do sofrimento psicológico. A Enfermagem tem, portanto, a responsabilidade fundamental de reconhecer o seu papel na manutenção dos cuidados paliativos em pacientes terminais. As estratégias de cuidados paliativos devem ser individuais, centradas no paciente, estabelecendo comunicação com a família, visando o cuidado integral (WHO,2017).

O enfermeiro, integrante da equipe multidisciplinar, deve proporcionar uma abordagem terapêutica de qualidade no tratamento ao paciente terminal acometido pela DA, trabalhando com dignidade e respeito, ampliando a integralidade do cuidado (HENDRIKS SA, et al.,2014). Desta forma, a equipe de enfermagem deve adotar estratégias para promover uma boa comunicação, sejam elas a verbal e não verbal para subsidiar o cuidar humanizado ao paciente, de forma a proporcionar apoio, segurança, confiança, transmitir força e esperança, o que é primordial no contexto dos cuidados paliativos (MITCHELL SL, 2015).

Segundo a revisão realizada, a assistência de enfermagem deve ser planejada com antecedência, proporcionando conforto, avaliando a nutrição, observando se houve complicações de saúde, se há angústia e alteração de sintomas comportamentais, incluindo ajuda aos familiares nos dilemas éticos, e nas tomadas de decisão (MITCHELL SL, 2015); devem ser considerados os aspectos psicológicos e espirituais na assistência ao paciente e o atendimento de suas necessidades básicas afetadas (VOLICER L e SIMARD J, 2015); na assistência é imprescindível o conforto do paciente com doença terminal, e o controle de possíveis sintomas depressivos tornando a assistência ainda mais valorizada e eficiente, oferecendo qualidade de vida ao paciente e a família; monitorar os sinais não verbais de dor, que comprometem as atividades da vida diária (QUEIROZ RB, et al., 2014); preocupação com o conforto e dor do paciente, educação em saúde para beneficiar a prestação de cuidados paliativos para pessoas com demência (RAYMOND M, et al., 2014).

\section{Alívio do sofrimento e da dor para melhorar a qualidade de vida}

Segundo a OMS, "os cuidados paliativos são uma abordagem que melhora a qualidade de vida de pacientes e suas famílias que enfrentam doenças com risco de vida, através da prevenção e alívio do sofrimento por meio de identificação precoce, avaliação e tratamento da dor e outros problemas físicos, psicossocial e espiritual' (WHO, 2017). 
Embora a dor não seja uma consequência usual da demência, os pacientes com DA devem ser cuidadosamente monitorados quanto à presença de dor. Devido a déficits de memória pessoas com demência são menos propensas a relatar dor e, quando desenvolvem afasia, podem não poder informar verbalmente a presença de dor. De forma que gritar ou rejeitar o cuidado pode ser a maneira de comunicar a dor ou desconforto. Portanto, é importante monitorar sinais não-verbais de dor (VOLICER, 2009).

Neste contexto, outra prática contemplada nos cuidados paliativos é a sedação paliativa, que é a administração deliberada de fármacos que reduzem o nível de consciência, com o consentimento do paciente ou de seu responsável, que tem como objetivo o alívio dos sintomas refratários, que podem ser físicos como a dor, dispneia, hemorragias e sangramentos maciços, sejam eles emocionais como angústia e sofrimento existencial intenso e intratável. Cada paciente deve receber sedativo e dose adequados para paliar o seu sintoma refratário específico (VOLICER L e SIMARD J, 2015; JOHNSON C, 2017).

A dor mal controlada, não reconhecida e subtratada causa impacto, além do âmbito físico, incluindo também comprometimento das atividades da vida diária (AVD) e humor, comportamento inadequado como agitação e aflição, resistência ao cuidado, retraimento social, processo de pensamento anormal e delírios (QUEIROZ RB, et al., 2014).

Em relação ao alívio da dor e outros sintomas físicos que interferem na qualidade de vida do paciente com DA, verificamos no nosso estudo que todos os artigos descreveram a importância do alivio da dor: os cuidados paliativos devem promover intervenções e tratamento para sintomas angustiantes para melhoria da qualidade de vida do paciente (MITCHELL SL, 2015); monitorar sinais não verbais de dor (VOLICER L e SIMARD J, 2015); o controle e o alívio da dor são um direito do indivíduo e um dever dos profissionais, que devem criar estratégias para diminuir o sofrimento (QUEIROZ RB, et al.,2014); uso medidas não-farmacológicas para o alívio da dor (fisioterapia, terapêutica ocupacional, neuro-estimulação eléctrica, massagem) (HENDRIKS SA,et al.,2014); os cuidados paliativos em toda a trajetória da doença melhoram a qualidade de vida e aliviam o sofrimento (YEAMAN PA, et al.,2013; JOHNSON C, 2017).

\section{Importância da abordagem religiosa e espiritual em Cuidados Paliativos}

A espiritualidade é o refúgio de famílias e pacientes em estado terminal, que necessitam do cuidado paliativo não só para o alívio das dores físicas, mas das dores emocionais e relacionamentos envolvidos no processo do cuidado. Por conseguinte, é evidente uma intrínseca relação entre o cuidado paliativo e a espiritualidade, e como esses dois pilares são essenciais à qualidade de vida do paciente, especialmente ao terminal. Dessa forma, é importante que a equipe multidisciplinar compreenda e seja sensível às necessidades espirituais do paciente para promover o cuidado integralizado, que por vezes é ignorado pelas diretrizes curriculares no modelo cartesiano Flexneriano (SOUZA VCT, et al., 2012).

Neste contexto, é fundamental que a equipe de saúde, que assiste pacientes em cuidados paliativos, tenha conhecimento de suas próprias questões sobre finitude e espiritualidade, sendo o ponto vital nos cuidados com aqueles que estão partindo, porque a natureza religiosa e espiritual do ser humano é ainda pouco abordada pelos profissionais, sendo portanto crucial que a equipe aceite os diferentes valores religiosos e espirituais, sem julgamentos, permitindo a participação do paciente e familiares nos planos de cuidados (SAPORETTI LA e SILVA AMOP, 2012).

$\mathrm{Na}$ revisão realizada, verificamos que apenas dois artigos descrevem a importância dos aspectos psicológicos e espirituais da assistência ao paciente. Volicer L e Simard (2015) ressaltam a importância de prover assistência psicológica e espiritual de forma a melhorar a qualidade de vida, proporcionando apoio emocional ao paciente; e Queiroz RB et al. (2014) que enfatizam a necessidade de proporcionar suporte psicossocial e espiritual promovendo conforto e bem-estar do paciente.

\section{CONSIDERAÇÕES FINAIS}

A partir dos artigos analisados, conclui-se que os princípios básicos para os cuidados paliativos integrais compreendem reconhecer a morte como um processo natural da vida e incorporar a integração dos cuidados 
físicos, espirituais, emocionais e sociais na promoção do conforto dos pacientes. Conforme recomenda a filosofia paliativista, que compreende o paciente e sua família na sua subjetividade, a equipe de enfermagem deve atender as necessidades do paciente com doença de Alzheimer oferecendo cuidados de forma integral e humanizado, permitindo a participação da elaboração dos planos de cuidados, desenvolvendo uma relação de empatia baseada na dignidade e respeito de forma a auxiliá-los no processo de morte.

\section{REFERÊNCIAS}

1. ALZHEIMER'S ASSOCIATION. 2018 Alzheimer's disease facts and figures. Alzheimer's \& Dementia. $2018 ; 14$ (3):367-429.

2. ARAÚJO MMT, SILVA MJP. A comunicação com o paciente em cuidados paliativos: valorizando a alegria e o otimismo. Rev da Esc Enferm da USP. 2007;41(4):668-674.

3. BRASIL, Ministério da Saúde. Resolução no 41, de 31 de outubro de 2018. Dispõe sobre as diretrizes para a organização dos cuidados paliativos, à luz dos cuidados continuados integrados, no âmbito Sistema Único de Saúde (SUS). Diário Oficial da União, Brasília, DF, 23 nov. 2018. Seção 1, p 276.

4. DENING KH, et al. Preferences for end-of-life care: a nominal group study ofpeople with dementia and their family carers. Palliative Medicine,2013; 27, 409-417

5. CARDOSO DH, et al. Cuidados paliativos na assistência hospitalar: a vivência de uma equipe multiprofissional. Texto \& Contexto - Enfermagem, 22(4), 1134-1141

6. FIRMINO F. Pacientes portadores de feridas neoplásicas em serviços de cuidados paliativos: contribuições para a elaboração de protocolos de intervenção de enfermagem. Rev Bras Cancerol. 2005; 51(4):347-359.

7. HENDRIKS SA, et al. Dying With Dementia: Symptoms, Treatment, and Quality of Life in the Last Week of Life. J Pain Symptom Manage.2014; 47(4):710-720.

8. JOHNSON C. Living with dignity: a palliative approach to care at the end of life. ANMJ. 2017; 25:30-33.

9. MITCHELL SL. Advanced Dementia. N Engl J Med. 2015;373(13):1275-1277.

10. MOHER D, et al. PRISMA Group. Preferred reporting items for systematic reviews and meta-analyses: the PRISMA statement. Ann Intern Med. 2009; 151(4):264-269

11. NATIONAL INSTITUTES OF HEALTH-NIH, NATIONAL INSTITUTE ON AGING-NIA. What Happens to the Brain in Alzheimer's Disease? Disponível em: https://www.nia.nih.gov/health/what-happens-brain-alzheimers-disease. Acesso em: 26 out. 2019.

12. QUEIROZ RB, et al. Cuidados paliativos e Alzheimer: concepções de neurologistas. Rev Enferm UERJ. 2014;22(5):686-692.

13. RAYMOND M, et al. Palliative and end of life care for people with dementia: lessons for clinical commissioners. Prim Health Care Res Dev. 2014;15:406-417.

14. SANTOS FH, et al. Highlights from the International Summit on Intellectual Disability and Dementia Implications for Brazil. Dementia \& Neuropsychologia, 2018;12(4), 329-336.

15. SAPORETTI LA, SILVA AMOP. Importância da abordagem religiosa e espiritual em Cuidados Paliativos. In: Academia Nacional de Cuidados Paliativos, organizador. Manual de cuidados paliativos. Rio de Janeiro: Diagraphic. 2012 ; p. 556-559.

16. SILVA RS, et al. Cuidando do paciente no processo de morte na Unidade de Terapia Intensiva. Rev Esc Enferm USP. $2011 ; 45(3): 738-744$.

17. SOUZA VCT, et al. Bioética, religião, espiritualidade e a arte do cuidar na relação médico-paciente. Revista Bioethikos, Centro Universitário São Camilo - 2012;6(2):181-190

18. VOLICER, L. Do we need another dementia pain scale? J Am Med Dir Assoc. 2009 Sep;10(7):450-452.

19. VOLICER L, SIMARD J. Palliative care and quality of life for people with dementia: medical and psychosocial interventions. Int Psychogeriatrics. 2015;27(10):1623-1634.

20. WORLD HEALTH ORGANIZATION-WHO. World report on ageing and health. Geneva: WHO; 2015.

21. WORLD HEALTH ORGANIZATION-WHO. Palliative Care. Geneva: WHO; 2017.

22. YEAMAN PA, et al. Providing quality palliative care in end-stage Alzheimer Disease. Am J Hosp Palliat Care. 2013 Aug;30(5):499-502. 